\title{
Mortalidade neonatal precoce em um hospital terciário do nordeste brasileiro
}

\author{
Early neonatal mortality in a tertiary hospital of northeast Brazil
}

Mortalidad neonatal temprana en un hospital terciario del noreste de Brasil

Ana Virgínia Ferreira de Morais ${ }^{1}$ (DD https://orcid.org/0000-0003-2473-1337

Ana Maria Martins Pereira ${ }^{1}$ (D) https://orcid.org/0000-0003-2885-3075

\section{Resumo}

0 objetivo deste estudo foi investigar quais fatores influenciam na mortalidade neonatal precoce, identificando as causas mais prevalentes dos óbitos neonatais e descrevendo as mortes com potencial de evitabilidade. Trata-se de um estudo de análise documental, retrospectivo. Do total de 386 óbitos, ocorridos entre os anos de 2014 e 2017, 68,7\% desses ocorreram no período neonatal precoce. Com relação às causas dos óbitos, as variáveis encontradas no modelo hierárquico foram: Prematuridade/Prematuridade Extrema (74\%), Síndrome do Desconforto Respiratório (SDR)/Insuficiência Respiratória (36\%), Infecção Neonatal/Sepse (26,7\%) e Anóxia $(22,8 \%)$. Quanto à evitabilidade dos óbitos registrados, $59 \%$ foram classificados como evitáveis, tendo um aumento gradual com o passar dos anos. Dos óbitos evitáveis, $86,2 \%$ poderiam ser reduzíveis por adequada atenção às mulheres na gestação. Percebe-se que a maioria dos óbitos evitáveis tem relação direta com a assistência às gestantes no pré-natal e no parto.

\section{Abstract}

The objective of this study was to investigate which factors influence early neonatal mortality, identifying the most prevalent causes of neonatal deaths and describing deaths with avoidable potential. It is a documental analysis of retrospective study. Of the 386 deaths that occurred between 2014 and $2017,68.7 \%$ occurred in the precocious neonatal period. Regarding the causes of death, the variables found in the hierarchical model were: Prematurity / Extreme Prematurity (74\%), Respiratory Distress Syndrome / Respiratory Failure (36\%), Neonatal Infection / Sepsis (26.7\%) and Anoxia (22.8\%). As for the avoidability of deaths registered $59 \%$ were classified as preventable, having a gradual increase over the years. Of avoidable deaths, $86.2 \%$ could be reduced by adequate attention to women during pregnancy. Most avoidable deaths are directly related to prenatal and delivery care to pregnant women.

\section{Resumen}

El objetivo de este estudio fue investigar qué factores influyen en la mortalidad neonatal temprana, identificando las causas más prevalentes de muertes neonatales y describiendo aquellas muertes con potencial evitable. Es un análisis documental estudio retrospectivo. De las 386 muertes que ocurrieron entre 2014 y 2017, 68.7\% ocurrieron en el período neonatal temprano. En cuanto a las causas de muerte, las variables encontradas en el modelo jerárquico fueron: Prematuridad / Prematuridad extrema (74\%), Síndrome de dificultad respiratoria / Insuficiencia respiratoria (36\%), Infección neonatal / Sepsis (26.7\%) y Anoxia (22,8\%). En cuanto a la evitación de muertes registradas, el 59\% fueron clasificadas como prevenibles, con un aumento gradual a lo largo de los años. De las muertes prevenibles, el $86,2 \%$ podría reducirse mediante la atención adecuada a las mujeres durante el embarazo. La mayoría de las muertes prevenibles están directamente relacionadas con la atención prenatal y del parto a mujeres embarazadas.

\section{Descritores}

Mortalidade neonatal; Causas da mortalidade neonatal; Enfermagem pediátrica; Óbitos neonatais evitáveis

\section{Keywords}

Neonatal mortality; Causes of neonatal mortality; Pediatric nursing; Avoidable neonatal deaths

\section{Descriptores}

Mortalidad neonatal; Causas de mortalidad neonatal; Enfermería pediátrica; Muertes neonatales evitables

\section{Como citar:}

Morais AV, Pereira AM. [Early neonatal mortality in a tertiary hospital of northeast Brazil]. Rev Soc Bras Enferm Ped. 2019;19(2):89-96. Portuguese

${ }^{1}$ Escola de Saúde Pública do Ceará, Fortaleza, CE, Brasil.

Conflitos de interesse: nada a declarar.

Submetido: 30 de Setembro de 2019 | Aceito: 20 de Dezembro de 2019

Autor correspondente: Ana Virgínia Ferreira de Morais

E-mail: aninhacepb@gmail.com

Dol: http://dx.doi.org/10.31508/1676-3793201900012 


\section{Introdução}

Em todo o mundo a mortalidade infantil vem decrescendo, e o Brasil vem acompanhando o cenário mundial, entretanto, quando se fala em óbito neonatal (nos primeiros 27 dias de vida), o País ainda tem muito para melhorar, principalmente nas regiões Norte e Nordeste. ${ }^{(1)} \mathrm{A}$ redução da mortalidade neonatal tem sido mais lenta do que a redução da mortalidade para as crianças entre um e cinquenta e nove meses, as mortes neonatais representam, agora, uma parcela maior das mortes totais abaixo dos cinco anos. Todas as regiões do mundo verificam um aumento na proporção das mortes abaixo dos cinco anos que ocorrem no período neonatal. ${ }^{(2)}$

No Brasil, a situação não é diferente, segundo dados da Pesquisa Nascer no Brasil, a mortalidade neonatal vem sendo o principal componente da mortalidade infantil desde a década de 1990 no País e vem se mantendo em níveis elevados. ${ }^{(3)} \mathrm{Na}$ década de 80 , a porcentagem de óbitos neonatais ficava entre $54 \%$ e $70 \%$ do total da mortalidade infantil, todavia na segunda metade da década de 90, passou a representar mais de $60 \%$ das mortes na infância. Além disso, mais de $70 \%$ desses óbitos ocorrem no período neonatal precoce, com maior prevalência nas primeiras 24 horas de vida. ${ }^{(4)}$

O coeficiente de mortalidade neonatal é um indicador negativo que está intimamente relacionado à assistência recebida pelas mulheres no período antenatal, parto e neonatal, refletindo a qualidade do atendimento prestado. ${ }^{(5)}$

A redução da mortalidade infantil neonatal é ainda mais difícil, pois está associada tanto a fatores biológicos quanto a assistência pré-natal, ao parto e ao recém-nascido; e sua prevenção envolve, principalmente, investimentos em serviços hospitalares de tecnologia mais complexa, bem como em ações educativas e de saúde pública. ${ }^{5,6)}$

Algumas estratégias criadas pelo Ministério da Saúde buscam dar apoio à saúde da criança de forma integral, tais como: a Atenção Humanizada ao Recém-Nascido de Baixo Peso - Método Canguru e a Rede Cegonha, entre outros. ${ }^{(7)}$

A Rede Cegonha, instituída através da Portaria no 1.459, de 24 de junho 2011, consiste numa rede de cuidados que visa assegurar à mulher o direito ao planejamento reprodutivo e à atenção humanizada à gravidez, ao parto e ao puerpério, assim como à criança o direito ao nascimento seguro, ao crescimento e ao desenvolvimento saudáveis. Um dos objetivos do Programa Rede Cegonha é erradicar os altos índices de mortalidade materna no Brasil e diminuir taxas de mortalidade infantil através de um novo modelo de atenção ao parto no Brasil. ${ }^{(8)}$

Há 15 anos a Atenção Humanizada ao Recém-Nascido de Baixo Peso - Método Canguru, com abrangência nacional e reconhecimento internacional, através de práticas clínicas e evidências científicas, vem melhorando a assistência ao recém-nascido de baixo peso e suas famílias, garantindo a humanização do cuidado neonatal, compreendida como respeito à integralidade e à singularidade de cada recém-nascido, não dissociada da qualidade técnico-científica e das boas práticas do campo da terapia intensiva neonatal. ${ }^{(9)}$

No Ceará, a situação é semelhante ao contexto nacional e a mortalidade infantil vem sendo tratada como prioridade nesse Estado. A vigilância epidemiológica dos óbitos, através da formação dos Comitês de Prevenção à Mortalidade Materno Infantil, tem se mostrado uma importante ferramenta para alcançar as metas pactuadas para redução da mortalidade infantil através da investigação e da avaliação das causas dos óbitos, propondo medidas de intervenção para sua diminuição. ${ }^{(10)}$

O conceito de morte evitável surgiu na década de 1970, nos Estados Unidos das Américas, quando Rutstein na Universidade de Harvard se referia àqueles óbitos que poderiam ser evitados por serviços de saúde efetivos através da prevenção e/ou tratamento do evento ou condição que contribuiu para sua ocorrência. ${ }^{(1)}$

Foram elaborados vários sistemas de classificação para a análise da evitabilidade dos óbitos infantis. A lista de causas de mortes evitáveis por intervenções, no âmbito do SUS, foi elaborada pelo Ministério da Saúde, com o intuito de avaliar a qualidade dos serviços de saúde. Ela vem sendo utilizada como ferramenta de medição do grau de excelência da atenção à saúde. ${ }^{(11)}$ Os óbitos infantis evitáveis são aqueles que, por interferência de uma boa assistência prestada nos serviços de saúde, poderiam ter sido diminuídos. Sendo assim, um número elevado desse tipo de óbito indica falha na atenção à saúde. ${ }^{(5)}$

A Lista de causas de mortes evitáveis por intervenções, no âmbito do SUS, classifica as causas de 
morte como: evitáveis, não evitáveis, mal definidas e não classificadas. As mortes por causas evitáveis são divididas em categorias; reduzíveis por ações de imunoprevenção, reduzíveis por adequada atenção à mulher na gestação, reduzíveis por adequada atenção à mulher no parto, reduzíveis por ações adequadas de diagnóstico e tratamento, reduzíveis por ações adequadas de promoção à saúde, vinculadas a ações adequadas de atenção à saúde. ${ }^{(10)}$

A mortalidade neonatal é um dos desafios para atingir o $4^{\circ}$ objetivo do milênio na assistência perinatal. Diminuir os números desse indicador tem sido o debate constante em todo o mundo e no Brasil, no intuito de proporcionar melhorias na assistência materno-infantil. A identificação dos principais grupos e causas de morte e seu potencial de redução e evitabilidade reflete a importância de reconhecer ações bem-sucedidas e lacunas ainda existentes no processo de trabalho e na organização dos serviços de saúde voltadas para a atenção de mulheres e crianças. ${ }^{(2)}$

O presente estudo torna-se relevante, uma vez que possibilita, em um hospital terciário, cujo público alvo são gestantes e recém-nascidos de alto risco, a análise do panorama da mortalidade neonatal, tal como repensar a assistência prestada e fornecer subsídios para implantação de intervenções mais apropriadas que garantam uma assistência de qualidade. A participação da enfermagem pediátrica assume um papel de extrema relevância nesse cenário, conduzindo para uma prática voltada para a saúde reprodutiva e neonatal sob um enfoque epidemiológico e de gênero, exigindo assim, uma nova postura ética na construção coletiva das práticas e saberes.

Diante do exposto, objetivou-se investigar quais os fatores que influenciam na mortalidade neonatal, tendo como base as informações disponíveis nos registros do Comitê de Prevenção à Mortalidade Materna Infantil em um hospital Público de Fortaleza, de acordo com as investigações dos óbitos e identificar as causas mais prevalentes dos óbitos neonatais na instituição, descrevendo as mortes com potencial de evitabilidade.

\section{Métodos}

Trata-se de um estudo de análise documental, retrospectivo, segundo $\mathrm{Gil}^{\left({ }^{(12)}\right.}$ esta forma de pesquisa vale-se de materiais que não receberam ainda um tratamento analítico, ou que ainda podem ser reelaborados de acordo com os objetos da pesquisa. ${ }^{(12)}$ Além de analisar os documentos de "primeira mão", existem também aqueles que já foram processados, mas podem receber outras interpretações. As fontes para coleta de dados foram as fichas de investigação de óbitos infantis analisadas pelo Comitê de Prevenção da Mortalidade Materna e Infantil local.

O estudo foi realizado em um Hospital Terciário, no município de Fortaleza, referência materno infantil no Estado e referência no Nordeste no Método Canguru. Foram utilizadas as fichas de investigação de óbitos neonatais ocorridos nos anos de 2014 a 2107 que foram analisadas e concluídas pelo Comitê de Prevenção à Mortalidade Materna Infantil local. A portaria nº 72/SVS/MS de 11 de janeiro de 2010, estabelece que a vigilância do óbito infantil e fetal é obrigatória nos serviços de saúde (públicos e privados) que integram o Sistema Único de Saúde. ${ }^{(13)}$

Foram utilizados os seguintes critérios de inclusão: os óbitos neonatais precoces, ocorridos entre os anos de 2014 a 2017, investigados pelo Comitê de Prevenção da Mortalidade Materna e Infantil, tendo como critério de exclusão, todos os óbitos neonatais precoces que não tiveram sua investigação concluída pelo comitê.

Foi construído um instrumento de coleta, baseado na ficha de investigação de óbitos infantis, para agrupamento dos dados, que foram coletados no setor de epidemiologia da instituição pesquisada no período de junho a agosto de 2018 pelo próprio pesquisador. Utilizou-se das causas dos óbitos registradas nas declarações de óbitos em qualquer linha, cada investigação tem uma cópia dessas em anexo. Assim como para a classificação da evitabilidade, fatores intervenientes e recomendações, foram usadas as que já existiam na ficha de investigação feita pelo comitê, que utiliza da Lista de Causas de Mortes Evitáveis por Intervenções, no âmbito do SUS. Já os dados referentes ao Estado foram colhidos no SIM e SINASC. ${ }^{(14,15)}$

Outros dados colhidos foram a idade gestacional ao nascimento para classificação em pré-termo, termo e pós-termo, a idade no momento do óbito para classificação enquanto óbito neonatal (precoce e tardio) e pós-neonatal.

Os dados foram organizados em uma planilha no programa Excel 2013, sendo agrupados por ano. Pos- 
teriormente ao agrupamento, os dados foram analisados por meio da estatística descritiva e apresentados no presente estudo em forma de gráficos e percentuais.

O projeto de pesquisa foi aprovado pelo Comitê de Ética e Pesquisa do Hospital Geral Dr. César Cals/ SES/SUS sob parecer consubstanciado $\mathrm{n}^{\circ} 2.644 .878$, Certificado de Apresentação ara Certificação Ética (CAAE) n 88802318.2.0000.5041. A instituição assinou um Termo de Fiel Depositário de modo a autorizar a realização da pesquisa.

\section{Resultados}

A figura 1 mostra a distribuição de óbitos em menores de um ano, ocorridos entre os anos de 2014 a 2017, um total de 562 óbitos, onde a maior frequência, ou seja, $68,7 \%$ desses, ocorridos entre os primeiros seis dias de vida (óbito neonatal precoce). Entre o período neonatal tardio e pós-neonatal, a frequência de óbitos foi respectivamente $18,3 \%$ e $13 \%$, evidenciando, assim, que a menor ocorrência de óbitos infantis, na instituição pesquisada, prevaleceu no período pós-neonatal.

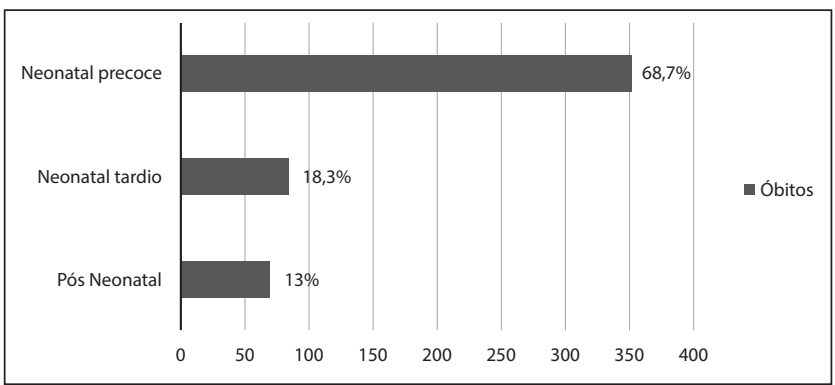

Figura 1. Distribuição dos óbitos infantis por componente etário

Com relação às causas dos óbitos, as variáveis que se mostraram associadas ao óbito neonatal precoce no modelo hierárquico foram: prematuridade/prematuridade extrema em $74 \%$, Síndrome do Desconforto Respiratório(SDR)/Insuficiência Respiratória em 36\%, Infecção Neonatal/Sepse em 26,7\% e Anóxia em 22,8\% (Figura 2).

A figura 3 evidencia a maior e menor taxa de óbitos neonatais precoces respectivamente em pré-termo (28 a 36s) $96 \%$ e termos ( $\geq 37 \mathrm{~s}$ ) $4 \%$, dentre os pré-termos, $65 \%$ deles eram pré-termos extremos $(<28 \mathrm{~s})$.

Quanto à evitabilidade dos óbitos neonatais precoces registrados entre os anos de 2014 a 2017, 227 (59\%)

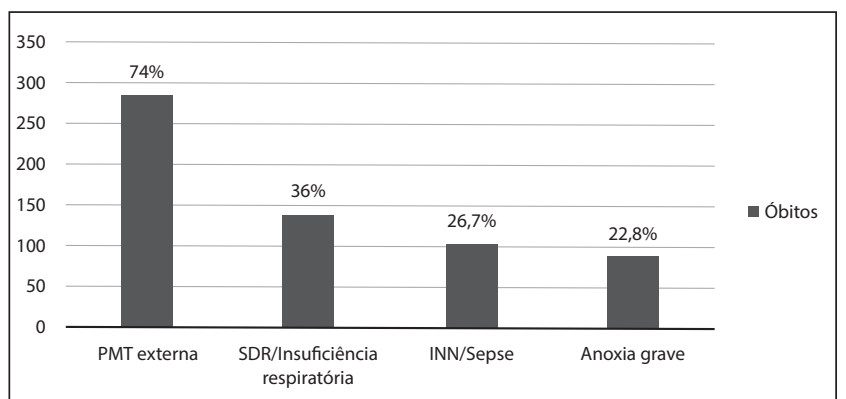

Figura 2. Mortalidade neonatal precoce e causas dos óbitos

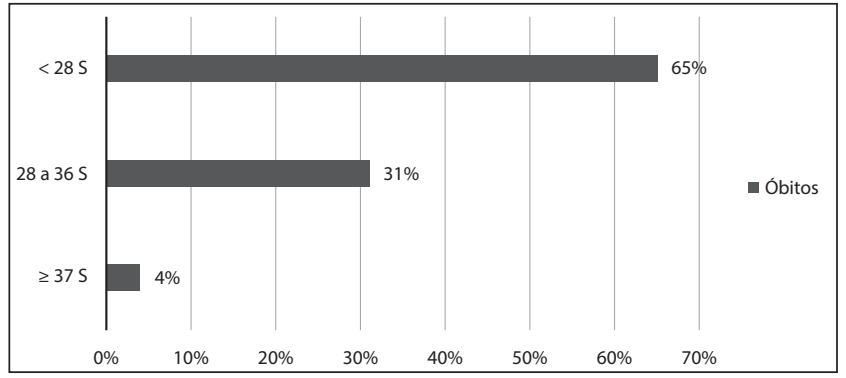

Figura 3. Óbito neonatal precoce por componente etário

foram classificados como evitáveis, 157 (41\%) como não evitáveis e $2(0,5 \%)$ como mal definidos, tendo um aumento gradual com o passar dos anos (Figura 4).

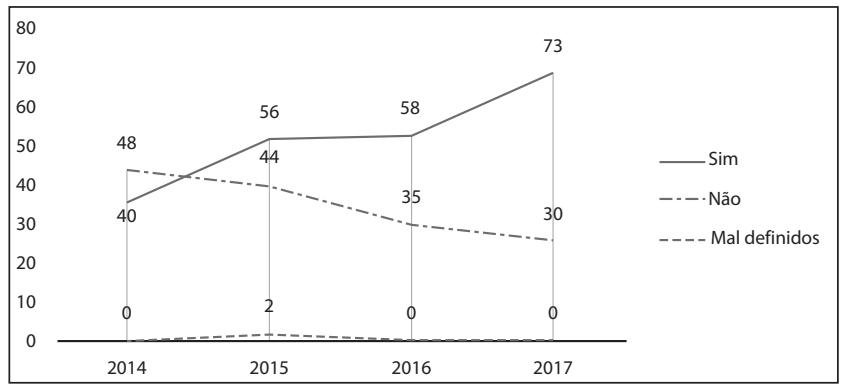

Figura 4. Proporção de óbitos neonatal precoce segundo critério de evitabilidade

As causas evitáveis foram classificadas pelo Comitê local, utilizando-se a lista de causas de mortes evitáveis por intervenções, no âmbito do SUS. Dos 210 óbitos evitáveis, $86,2 \%$ poderiam ser reduzíveis por adequada atenção a mulher na gestação (Figura 5).

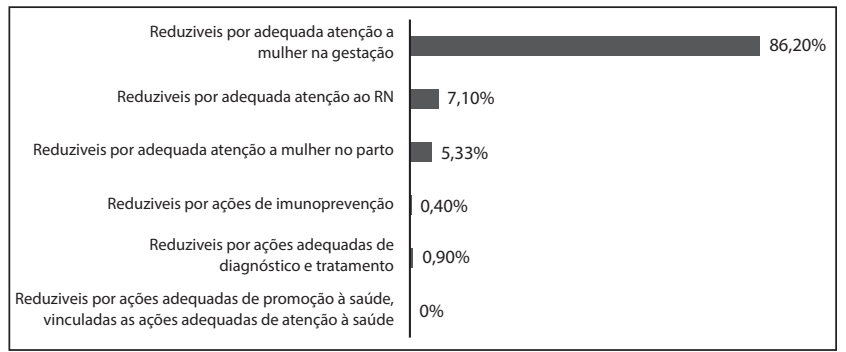

Lista brasileira de causas de mortes evitáveis por intervenções do Sistema Único de Saúde-SUS.

Figura 5. Critério de evitabilidade 


\section{Discussão}

A mortalidade neonatal representa mais de dois terços dos óbitos no primeiro ano de vida e tem sido amplamente discutida, sobretudo pela dificuldade de redução e, ainda, por requerer medidas de alto custo e complexidade para melhoria da qualidade na assistência pré e perinatal. ${ }^{(4)} \mathrm{O}$ aumento dos óbitos neonatais em detrimento da redução dos pós-neonatais é semelhante em todo o mundo, e desde a década de 80 , passou a representar mais de $70 \%$ da mortalidade infantil no Brasil. ${ }^{(4,16)}$

No Ceará, de acordo com os dados do SIM (Sistema de Informações sobre Mortalidade, entre os anos de 2013 a 2017, foram registrados 5.811 óbitos neonatais, dos quais 4.506 neonatais precoces, representando estes $77,5 \%$ dos óbitos neonatais e $54,73 \%$ da mortalidade infantil do Estado, evidenciando o peso expressivo da mortalidade neonatal precoce.

$\mathrm{Na}$ instituição pesquisada, constatou-se que elevada proporção $(68,7 \%)$ dos óbitos, entre os anos de 2014 a 2017, ocorreu no período neonatal precoce, e a menor proporção (13\%) no período pós-neonatal, tal como se vem verificando no Estado e no Brasil.

O componente pós-neonatal predominou no Brasil até o final da década de 80; a partir de então prevalece o componente neonatal. Nos países pobres da África, no entanto, os óbitos neonatais representam pouco mais de $30 \%$ da mortalidade infantil, tendo em vista as condições desfavoráveis de vida e saúde da população que elevam a mortalidade pós-neonatal. ${ }^{(4)}$

A redução da mortalidade infantil tem sido atribuída a diversos fatores, como a queda da fecundidade, maior acesso da população ao saneamento e serviços de saúde, aumento da prevalência de aleitamento materno, às ações de imunização, antibioticoterapia e terapia de reidratação oral, entre outras. ${ }^{(6)}$

Com relação às causas dos óbitos, constava nas declarações de óbitos, em qualquer linha, hierarquicamente: 74\% - Prematuridade/Imaturidade (P07-Transtorno Relacionado a Gestação de Curta Duração com Peso Baixo ao Nascer), 36\% - Síndrome do Desconforto Respiratório(SDR)/Insuficiência Respiratória (P22 - Desconforto Respiratório do Recém-nascido), 26,7\% Infecção Neonatal/ Sepse (P36 - Septicemia Bacteriana do Recém-nascido) e 22,8\% - Anóxia (P21 - Asfixia ao nascer). Esses dados se reafirmam, quando compara- dos aos encontrados no TabNet Win, ${ }^{(14)}$ entre os anos de 2013 a 2017, com relação as principais causas de óbitos na instituição pesquisada.

As principais causas de morte neonatal precoce, a imaturidade e o desconforto respiratório do recém-nascido, observadas neste trabalho, estariam relacionadas às falhas no manejo obstétrico e/ou da reanimação neonatal. Isso indica deficiências na assistência pré-natal, ao parto e ao recém-nascido, relacionadas à qualidade de atenção nos serviços de saúde. Algumas variáveis como número de consultas de pré-natal, detecção e consequente tratamento das intercorrências gestacionais seriam dados que poderiam estar associados a essas causas, porém não foram avaliados nessa pesquisa.

Segundo estudo de coorte sobre a mortalidade neonatal na pesquisa Nascer no Brasil,(3) a causa de morte neonatal mais comum foi a prematuridade e suas complicações (1/3 dos casos), seguida pela malformação congênita $(22,8 \%)$, as infecções $(18,5 \%)$, os fatores maternos $(10,4 \%)$ e asfixia/hipóxia $(7 \%){ }^{(6)}$ Compreender melhor essas causas e o que determina a mortalidade neonatal no Brasil representa, portanto, um passo importante na análise da saúde infantil para a definição das políticas públicas prioritárias.

Entende-se por prematuridade ou nascimento pré-termo todo nascimento ocorrido antes de 37 semanas de idade gestacional e esse nascimento antecipado é a principal causa dos óbitos infantis ocorridos na primeira semana de vida no Brasil e em todas as regiões, com importância relativa maior no primeiro dia de vida. ${ }^{18)}$

As causas da prematuridade são multifatoriais e envolvem, entre outros, fatores de risco que variam desde raça/etnia, gemelaridade, idade materna menor que 16 e maior que 40 anos, uso de tabaco, álcool e outras drogas, comorbidades maternas, partos prematuros anteriores determinadas infecções na gravidez até a realização de cesáreas eletivas com erro de data estimada. ${ }^{(19)}$

A prevenção de mortes decorrentes de complicações da prematuridade começa com o desenvolvimento de cuidados de qualidade antes, entre e durante as gravidezes, garantir gestações saudáveis é o primeiro passo. As diretrizes de cuidados pré-natais da $\mathrm{OMS}^{(20)}$ incluem intervenções-chave para ajudar a prevenir o nascimento prematuro, como assegurar uma alimen- 
tação saudável e nutrição ideal incluindo o uso de vitaminas pré-natais e ácido fólico, evitar o uso de tabaco e drogas ilícitas, assistência pré-natal com o mínimo de 8 contatos com profissionais de saúde, incluindo o uso de ultrassom para ajudar a determinar a idade gestacional e detectar gravidezes múltiplas bem como a identificação e gerenciamento de outros fatores de risco, como infecções. ${ }^{(20)}$

Para Oliveira ${ }^{(19)}$ é necessário um maior esforço das políticas de saúde pública para implementar medidas de assistência perinatal de modo articulado entre o programar e o executar permitindo uma abordagem integral e interdisciplinar dos vários níveis que compõem a atenção integral a gestante.

Ações para melhorar a assistência ao recém-nascido prematuro, incluem a melhor abordagem para a síndrome do desconforto respiratório (SDR) do recém-nascido ou Doença da Membrana Hialina (DMH), a qual acomete principalmente o recém-nascido pré-termo, pois decorre da deficiência do surfactante alveolar associada à imaturidade estrutural dos pulmões ${ }^{(7)}$. Essa patologia aparece nesse estudo, como a segunda causa de morte neonatal precoce mais frequente.

O uso antenatal de corticoide e a administração pós-natal de surfactante são medidas efetivas para prevenir e tratar a SDR, aliado aos avanços das técnicas ventilatórias alcançados nas últimas décadas. ${ }^{(21)}$

Corroborando com os autores, ${ }^{(21)}$ as recomendações da $\mathrm{OMS}^{(20)}$ trazem intervenções para melhorar o desfecho do nascimento de prematuros, entre elas está o uso de corticoide antenatal que deverá ser considerado para toda gestante com idade gestacional entre 24 e 34 semanas com risco de parto prematuro iminente para induzirem a produção de surfactantes, bem como o uso de surfactante, devendo o mesmo ser uma rotina na terapêutica do recém-nascido pré-termo com SDR e deverá ser instituída nas primeiras duas horas de vida, sendo reavaliada a necessidade de doses adicionais a cada seis horas.

Como visto, a implementação de práticas para minimizar a gravidade da insuficiência respiratória e a lesão pulmonar deve iniciar-se antes de o RN chegar à UTI, portanto, diante do nascimento de um recém-nascido pré-termo é fundamental a presença, na sala de parto, de uma equipe de profissionais com experiência em reanimação neonatal, para maiores detalhes sobre cuidado na sala de parto.
Outra causa de óbito neonatal precoce na instituição pesquisada é a Sepse Neonatal Precoce (que ocorre nos primeiros seis dias de vida), 26,7\% das declarações de óbitos apresentaram, em qualquer campo, diagnóstico médico de sepse neonatal.

A sepse neonatal precoce está relacionada diretamente a fatores maternos gestacionais e periparto. ${ }^{(22)} \mathrm{O}$ germe mais comumente encontrado é do trato genital materno. Como os sinais clínicos são mínimos ou pouco específicos, os fatores de risco para o desenvolvimento da infecção são os responsáveis pela escolha de tratamento empírico com antimicrobianos.

Destaca-se, então, a importância do acompanhamento pré-natal e o tratamento das afecções maternas como ação preventiva da sepse neonatal precoce.

De acordo com Freitas, Pereira e Oliveira ${ }^{(23)}$ a anóxia grave ou asfixia perinatal refere-se a privação de oxigênio (hipóxia) e/ou falta de perfusão (isquemia) em vários órgãos, com alterações bioquímicas e funcionais, ressaltando aqui o dano cerebral e aparece nessa pesquisa como a quarta causa de morte mais frequente na instituição pesquisada. Pesquisa realizada no Paraná aponta a hipóxia como causa mais frequente de morte em recém-nascidos. ${ }^{(24)}$

Segundo Takazono e Golin ${ }^{(25)}$ e Freitas, Pereira e Oliveira $^{(23)}$ corroboram quando afirmam que a maioria dos casos de asfixia perinatal é de origem intrauterina. Aproximadamente 5\% ocorrem antes do início do parto, $85 \%$ durante a fase de trabalho de parto e período expulsivo e $10 \%$ durante o período neonatal. Reforçando assim a necessidade de uma assistência qualificada a gestante e seu recém-nascido que se inicia no período pré-concepcional até o nascimento do bebê.

Com relação a evitabilidade, 58,5\% dos óbitos ocorridos entre 2014 a 2017 foram classificados como evitáveis, destacando-se que 194 (86,2\%) dos óbitos evitáveis poderiam ter sido reduzíveis por adequada assistência a mulher na gestação, 12 (5,3\%) por adequada atenção ao recém-nascido, $16(7,1 \%)$ por adequada atenção a mulher no parto, $2(0,9 \%)$ por ações adequadas de diagnóstico e tratamento e $1(0,4 \%)$ por ações de imunoprevenções.

Para Dias, Santos Neto e Andrade ${ }^{(26)}$, o emprego dos métodos de evitabilidade, consiste em um importante instrumento para o diagnóstico das falhas de desempenho dos serviços de saúde e a orientação de medidas para reduzir os óbitos infantis evitáveis. 
Os autores ${ }^{(27)}$ concordam com Dias, Santos e Andrade $^{(26)}$ quando dizem que a abordagem da evitabilidade auxilia nas discussões relacionadas a organização, qualidade e acesso aos serviços de saúde, bem como a identificação dos óbitos que poderiam ter sido prevenidos ou evitados por uma adequada atenção à saúde materno-infantil.

A alta prevalência dos óbitos evitáveis reduzíveis por adequada atenção a mulher na gestação observada nesse estudo alerta para necessidade de melhoria na assistência pré-natal e estruturação da rede cegonha, para garantir atenção de qualidade, integral e continua ao binômio mãe/filho.

Outra pesquisa, realizada em Curitiba, diferentemente desta, apontou o maior número de óbitos neonatais por causas evitáveis, aquelas reduzíveis por adequada atenção ao recém-nascido.(28)

\section{Conclusão}

Apesar da redução da proporção da mortalidade infantil nos últimos anos no Brasil, houve aumento da concentração da mortalidade neonatal precoce, o que aponta para a necessidade de um maior cuidado a atenção materno-infantil. Muito ainda se tem a aprimorar para gerar maiores efeitos de queda nos índices de mortalidade neonatal. Percebe-se que a maioria dos óbitos evitáveis tem relação direta com a assistência à gestante no pré-natal e no parto, demonstrando a necessidade de instituir uma prática efetiva e de qualidade. Os resultados evidenciados aliados aos dados encontrados na literatura reforçam a necessidade de investimento na ampliação da qualidade da assistência à saúde neonatal e em todo o processo gestacional, intensificando esforços na realização de ações de promoção da saúde e prevenção de doenças, visando a redução da exposição aos fatores de risco evitáveis que podem conduzir à prematuridade extrema e ao desfecho óbito.

A importância da participação da Enfermagem Pediátrica neste desafio, vem sendo reconhecida no plano legal e institucional para assumir um papel de liderança frente a execução das políticas e portarias do Ministério da Saúde que abrangem essa temática. Melhorias nas condições socioeconômicas da população, o monitoramento e fortalecimento da Rede
Cegonha, uma maior abrangência à saúde das gestantes, os progressos científicos e tecnológicos tanto no campo do diagnóstico pré-natal como no campo da Pediatria são elementos que possibilitam o refinamento no cuidado prestado às gestantes e aos recém-nascidos. A mortalidade neonatal adquire uma importância sem precedentes, pois as ações necessárias para o seu controle são ainda pouco sistematizadas e incipientes no âmbito nacional, demandando uma mobilização e priorização na agenda dos gestores da saúde. O presente estudo considera a necessidade de pesquisas na área que auxiliem na elaboração de estratégias visando a ampliação da qualidade do acesso, do cuidado e da assistência em saúde com vistas a reduzir a exposição de gestantes aos fatores de risco para a ocorrência do desfecho óbito neonatal.

\section{Colaborações}

Morais AVF e Pereira AMM declaram que contribuíram com a concepção do estudo, análise e interpretação dos dados, redação do artigo, revisão crítica relevante do conteúdo intelectual e aprovação da versão final a ser publicada.

\section{Referências}

1. Silva CF, Leite AJ, Almeida NM, Leon AC, Olofin I. Fatores associados ao óbito neonatal de recém-nascidos de alto risco: estudo multicêntrico em Unidades Neonatais de Alto Risco no Nordeste brasileiro. Cad Saúde Pública. 2014;30(2):355-68.

2. Organização das Nações Unidas. Relatório dos objetivos de desenvolvimento do milênio [Internet]. 2015 [cited 2020 Apr 15]. Available from: https://nacoesunidas.org/wpcontent/uploads/2015/07/MDG-2015-June-25.pdf

3. Leal MC, Gama SG. Nascer no Brasil [editorial]. Cad Saúde Pública. 2014;30(Suppl 1):S5.

4. Gaíva MA, Bittencourt RM, Fujimori E. Óbito neonatal precoce e tardio: perfil das mães e dos recém-nascidos. Rev Gaucha Enferm. 2013;34(4):91-7.

5. Nascimento SG, Oliveira CM, Sposito V, Ferreira DK, Bonfim CV. Mortalidade infantil por causas evitáveis em uma cidade do nordeste do Brasil. Rev Bras Enferm. 2014;67(2):20812.

6. Lansky S, Friche AA, Silva AA, Campos D, Bittencourt SD, Carvalho ML, et al. Pesquisa Nascer no Brasil: perfil da mortalidade neonatal e avaliação da assistência à gestante e ao recémnascido. Cad Saúde Pública. 2014;30(Suppl 1):S192-S207.

7. Brasil. Ministério da Saúde. Secretaria de Atenção à Saúde. Departamento de Ações Programáticas Estratégicas. Atenção à saúde do recém-nascido: guia para os profissionais de saúde. Brasília (DF): Ministério da Saúde; 2014.

8. Brasil. Ministério da Saúde. Portaria 1459/2011. Institui, no âmbito do Sistema Único de Saúde, a Rede Cegonha. Brasília (DF): Ministério da Saúde; 2011.

9. Brasil. Ministério da Saúde. Secretaria de Atenção à Saúde. Departamento de Ações Programáticas Estratégicas. Atenção humanizada ao recém-nascido: método canguru manual técnico. Brasília (DF): Ministério da Saúde; 2017. 
10. Ceará (Estado). Núcleo de Vigilância Epidemiológica e Coordenadoria de Promoção e Proteção à Saúde. Manual de normas técnicas para Vigilância Epidemiológica do Óbito Materno, Infantil, Fetal e com Causa Mal Definida [Internet]. Secretaria da Saúde do Estado do Ceará; 2010. [citado 2020 Abr 20].Disponível em: https://www.saude.ce.gov. br/wp-content/uploads/sites/9/2018/06/manual_obito_materno.pdf

11. Brasil. Ministério da Saúde. Secretaria de Vigilância em Saúde. Departamento de Análise de Situação de Saúde. Coordenação Geral de Informação e Análise Epidemiológica. Manual de vigilância do óbito infantil e fetal e do comitê de prevenção do óbito infantil e fetal. Brasilia (DF): Ministério da Saúde; 2009.

12. Gil AC. Como elaborar projetos de pesquisa. 4a ed. São Paulo: Atlas; 2008.

13. Brasil. Ministério da Saúde. Portaria 72/2010. Estabelece que a vigilância do óbito infantil e fetal é obrigatória nos senviços de saúde (públicos e privados) que integram o Sistema Único de Saúde. Brasília (DF): Ministério da Saúde; 2010.

14. Ministério da Saúde. Sistema de Informações sobre Mortalidade (SIM). DATASUS [Internet]. Óbitos por causas evitáveis em menores de 5 anos - Ceará. 2018 [cited 2018 Dec 10]. Available from: http://tabnet.datasus.gov.br/cgi/deftohtm.exe?sim/cnv/evita10ce.def

15. Ministério da Saúde. Sistema de Informações sobre Nascidos Vivos (SINASC). DATASUS [Internet]. Nascidos vivos - Ceará. 2018 [cited 2018 Dec 10]. Available from: http:// tabnet.datasus.gov.br/cgi/deftohtm.exe?sinasc/cnv/nvce.def

16. Cavalcante AN, Araújo MA, Lopes SV, Almeida TI,Almeida RL. Epidemiologia da mortalidade neonatal no Ceará no período de 2005-2015. Rev Bras Promoç Saúde. 2018;31(4):1-8.

17. REDE Interagencial de Informação para a Saude. Indicadores básicos para a saúde no Brasil: conceitos e aplicações. 2a ed. Braślia (DF): Organização Pan-Americana da Saude; 2008. 349p.

18. Agência Nacional de Vigilância Sanitária. Senviços de atenção materna e neonatal: segurança e qualidade. Brasilia: ANVISA; 2014. Disponível em: http://twixar.me/1Pdn
19. Oliveira GC. Análise do catch-up de crescimento de uma coorte de recém-nascidos prematuros [Dissertação]. Cuiabá (MT): Universidade Federal de Mato Grosso; 2015.

20. World Health Organization (WHO). Recomendações da OMS sobre cuidados pré-natais para uma experiência positiva na gravidez [Internet]. Geneva: WH0, 2016 [cited 2020 Apr 15]. Available from: https://cdn2.sph.harvard.edu/wp-content/uploads/sites/32/2016/11/ WHO-RHR-16.12-por.pdf

21. Sousa DS, Sousa Júnior AS, Santos AD, Melo EV, Lima SO, Almeida-Santos MA, et al. Morbidade em recém-nascidos prematuros de extremo baixo peso em unidade de terapia intensiva neonatal. Rev Bras Saúde Mater Infant. 2017;17(1):139-47.

22. Alves JB, Gabani FL, Ferrari RA, Tacla MT, Linck Júnior A. Sepse neonatal: mortalidade em município do sul do Brasil, 2000 A 2013. Rev Paul Pediatr. 2018;36(2):132-40.

23. Freitas ZM, Pereira CU, Oliveira DM. Avaliação do nível de consciência de recém-nascidos com asfixia neonatal. Pediatr Mod. 2013;49(10):419-26.

24. Simões MA, Pabis FC, Freitas AK, Watanabe PK, Kayano RM, Noronha L. Causas evitáveis de morte e fatores associados à sobrevida de recém-nascidos em um hospital universitário da cidade de Curitiba, Paraná, Brasil. J. Bras Patol Med Lab. 2016;52(5):338-44.

25. Takazono PS, Golin MO. Asfixia perinatal: repercussões neurológicas e detecção precoce. Rev Neurocienc. 2013;21(1):108-17.

26. Dias BA, Santos Neto Et, Andrade MA. Classificações de evitabilidade dos óbitos infantis: diferentes métodos, diferentes repercussões? Cad Saúde Pública. 2017;33(5):e00125916.

27. Nascimento RM, Leite AJ,Almeida NM, Almeida PC, Silva CF. Determinantes da mortalidade neonatal: estudo caso-controle em Fortaleza, Ceará, Brasil. Cad Saúde Pública. 2012;28(3):559-72.

28. Moreira KF, Bicalho BO, Santos LC,Amaral FM, Orfão NH, Cunha MP. Perfil e evitabilidade de óbito neonatal em um município da Amazônia legal. Cogitare Enferm. 2017;(22)2:e48950. 